\title{
Physical and morphological characterization of reactively magnetron sputtered TiN films
}

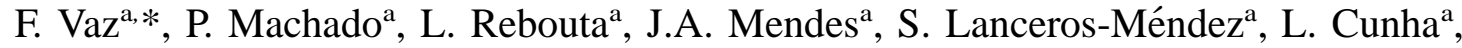 \\ S.M.C. Nascimento ${ }^{\mathrm{a}}$, Ph. Goudeau ${ }^{\mathrm{b}}$, J.P. Rivière ${ }^{\mathrm{b}}$, E. Alves ${ }^{\mathrm{c}}$, A. Sidor ${ }^{\mathrm{a}, 1}$ \\ an Universidade do Minho, Departamento de Física, Azurém, 4800-058 Guimarães, Portugal \\ ${ }^{\mathrm{b}}$ Université de Poitiers, Laboratoire de Métallurgie Physique, 86960 Futuroscope, France \\ 'ITN, Departamento de Física, E.N.10, 2685 Sacavém, Portugal
}

\begin{abstract}
The present paper reports the influence of growth conditions on the properties of TiN thin films deposited by rf reactive magnetron sputtering in the low-pressure range. The effects of rf power at the Ti target and the negative bias voltage at the substrate in the morphology, structure, electrical resistivity and colour of the samples were studied in detail. X-Ray diffraction results showed that the $\delta$-TiN phase $\left(a_{0} \sim 0.430 \mathrm{~nm}\right)$ is detected in all the samples. The sample prepared with grounded substrate revealed a lattice parameter close to the bulk value $(0.424 \mathrm{~nm})$, which is a consequence of a low stress state, due to the absence of ion bombardment. The sample deposited at $1000 \mathrm{~W}$ has a lattice parameter of $0.426 \mathrm{~nm}$, close to that of the stress-free material $\left(a_{0}=0.424 \mathrm{~nm}\right)$, probably due to some stress relief. All films have a columnar-type structure, lying in the T and I zone of the Thornton Model. The resistivity of the TiN films is almost constant and close to $60 \mu \Omega \mathrm{cm}$ independently of the preparation conditions, except for the films deposited at $1000 \mathrm{~W}, \rho \sim 215 \mu \Omega \mathrm{cm}$, and for the grounded sample, $\rho \sim 153 \mu \Omega \mathrm{cm}$. These values are probably due to cracks associated with stress relieves, in the first case, and the lack of ion bombardment that leads to films with lower density and higher number of defects in the second. No significant variations in colour were observed.
\end{abstract}

(C) 2002 Elsevier Science B.V. All rights reserved.

Keywords: Magnetron sputtering; TiN films; Rf power

\section{Introduction}

In the hard coating category, which ranges from hard carbon films to transition metal carbides, nitrides, borides, and silicides, titanium nitride (TiN) is, at present, one of the most studied and used materials [1]. TiN is chemically stable, highly resistant to corrosion by strong acids, and exhibits low friction and wear. Due to these properties, it is extensively used in a wide range of applications, from protective coating of machine parts and cutting tools [1] to application as diffusion barriers in semiconductor technology [2]. Moreover, TiN is also very popular as a decorative coating material for watchcases, watch bands, eye glass frames, door-handles, etc., due to its golden colour, although the exact matching of

\footnotetext{
*Corresponding author. Tel.: +351-25-351-0471; fax: +351-25351-0461.

E-mail address: fvaz@fisica.uminho.pt (F. Vaz).

${ }^{1}$ Formerly at Universidade do Minho, Dept. Física, Azurém, 4800058 Guimarães, Portugal.
}

hue and tone with that of gold is difficult due to the influence of both stoichiometry and microstructure of the final coating material $[3,4]$. TiN coatings are obtained by a variety of physical vapour deposition (PVD) and chemical vapour deposition (CVD) processes, such as activated reactive evaporation, ion plating using hollow cathode discharge (HCD), cathodic arc ion plating and sputter ion plating, and, most commonly, by $\mathrm{rf} / \mathrm{dc}$ reactive magnetron sputtering. The different processes give rise to a large scatter in the microstructures and properties of the films, and thus a lot of investigations are still being carried out in this material. Among these, a considerable number of studies have been performed in reactively sputtered $\mathrm{TiN}$ in order to investigate the relationship between deposition parameters, film structure and the final properties of the material. The correlation between the preferred orientation and the deposition conditions is an important issue for the applications of TiN coatings, since it has a significant effect on the material properties. Furthermore, it has been reported that in the absence of ion 
bombardment (provoked by a substrate bias), darkbrown films with relatively high resistivity are obtained [5-7]. When an appropriate negative bias is applied to the substrate, dense golden-coloured films with low resistivity are produced [6]. However, other authors claim that TiN films having gold colour and low resistivity can be obtained under a no-bias condition [8]. These discrepancies indicate that the dependence of film properties on the deposition conditions and the criterion for a particular film colour are still unclear and deserve further investigation $[9,10]$. Further, a systematic characterization of the structure and physical properties of TiN under different deposition conditions is essential for the search of new decorative coatings based on oxygen doping of $\mathrm{TiN}$, in which this team is involved [11].

In the present paper, the influence of rf power applied to the Ti target and dc negative biasing of the substrate on the deposition rate, morphology, microstructure, resistivity and colour of the TiN films will be addressed.

\section{Sample preparation and experimental techniques}

TiN films were deposited by reactive rf magnetron sputtering, from a high purity Ti target $(99.731 \%)$ onto polished high-speed steel (AISI M2), stainless steel, single crystalline silicon(100) and glass substrates. The depositions were carried out in an $\mathrm{Ar} / \mathrm{N}_{2}$ atmosphere using an Alcatel SCM650 apparatus. Depositions were carried out with the substrate statically placed over the target at $60 \mathrm{~mm}$ distance. Prior to all depositions, the substrates were ultrasonically cleaned and sputter etched for $15 \mathrm{~min}$ in a $0.4 \mathrm{~Pa} \mathrm{Ar}$ atmosphere (200 W rf power). Since the films prepared without an adhesion layer revealed extensive decohesion from the substrate due to the development of high compressive stresses, a pure titanium adhesion layer $(600 \mathrm{~W}$ rf power in Ti target, $T_{\mathrm{s}}=300{ }^{\circ} \mathrm{C}$ and $-50 \mathrm{~V}$ bias voltage), with a thickness of approximately $0.30 \mu \mathrm{m}$, was deposited in each sample, in order to improve the adhesion of the films to the substrates.

Two sets of samples were prepared: the first group was prepared with variation of the rf power applied to the Ti target, using constant values of temperature (300 $\left.{ }^{\circ} \mathrm{C}\right)$ and bias voltage $(-50 \mathrm{~V})$. Rf powers varied from 1.9 to $3.5 \mathrm{~W} / \mathrm{cm}^{2}$. Nitrogen partial pressure ranged from 0.02 to $0.05 \mathrm{~Pa}$. The second group was prepared with variation of the applied bias voltage and a fixed temperature $\left(300{ }^{\circ} \mathrm{C}\right)$ and a rf power of $1.9 \mathrm{~W} / \mathrm{cm}^{2}(600$ $\mathrm{W})$ applied to the Ti target. Both sets of samples were prepared with a constant argon flux of $100 \mathrm{sccm}$. The working pressure was approximately constant at $0.4 \mathrm{~Pa}$.

The stoichiometry of the deposited layers (50 at.\% of each of the two elements: Ti and N) was confirmed by Rutherford back scattering (RBS). No oxygen and argon were detected in the as-deposited samples, which means that their content is less than $\sim 2$ at.\%. The sample thickness was determined by an average of five ballcratering (BC) experiments and confirmed by scanning electron microscopy (SEM).

The morphology of the films was studied by SEM and optical microscopy (OM). X-Ray diffraction (XRD) experiments were used for structural characterization. XRD patterns were obtained using a conventional Philips PW1710 apparatus operated with $\mathrm{CuK} \alpha$ radiation. Although a detailed deconvolution was not performed, the average crystallite size (vertical coherence width) was determined by means of Fourier analysis. Since only one first order diffraction profile was available, the method introduced by Mignot and Rondot [12], based on the analysis of a single X-ray profile, was applied. The resistivity of the films was measured by the Van der Pauw method [13] at room temperature, using silver paint contacts in the films deposited in $\mathrm{Si}$ substrates.

The colour of the samples with enough clear area was computed from spectral data acquired using a hyperspectral imaging system. Samples were illuminated at $8^{\circ}$ by radiation from a Xenon lamp coupled with a tuneable birefringent filter (VariSpec, model VS-VIS210HC-35-SQ, Cambridge Research \& Instrumentation, Inc, USA), tuneable over the wavelength range 400$720 \mathrm{~nm}$ with a half-height full-width of $10 \mathrm{~nm}$ at 500 $\mathrm{nm}$. Specular reflected light was acquired with a highresolution monochrome digital camera (ORCA-ER, Hamamatsu, Japan), with spatial resolution of $1344 \times 1024$ pixels and 12-bit intensity resolution. Measurements were made against the white standard $\mathrm{BaSO}_{4}$ at $10 \mathrm{~nm}$ intervals. An area of $3.7 \times 3.7 \mathrm{~mm}$ was analysed with a spatial resolution of $12 \mu \mathrm{m} /$ pixel. The colour specification under the standard CIE illuminant $\mathrm{D}_{65}$ was computed and represented in the CIELAB 1976 colour space $[14,15]$ for each individual pixel in the area. Only the samples with enough available area were analysed.

\section{Results and discussion}

\subsection{Dependence on the rf power applied to the target}

The increase of rf power applied to the Ti target linearly increases the deposition rate, $R_{\mathrm{d}}$ (Fig. 1a). This change is due to the increase in the self-bias potential, which by its turn increases the ion bombardment energy as $e\left[V_{\mathrm{p}}-V_{\mathrm{sb}}\right]$ ( $V_{\mathrm{sb}}$ is the target self-bias; $V_{\mathrm{p}}$ is the plasma potential, approx. constant [16]) and thus the energy and number of ejected species.

$\mathrm{X}$-Ray diffraction experiments show that there is a unique TiN phase in all samples, $\delta$-TiN with fcc $\mathrm{NaCl}$ type lattice [17] (Fig. 2). Additional diffraction peaks are due to both steel substrates and the $\mathrm{Ti}$ adhesion (buffer) layer. These results seem to indicate a preferred orientation, with $\{111\}$ planes mostly parallel to the 


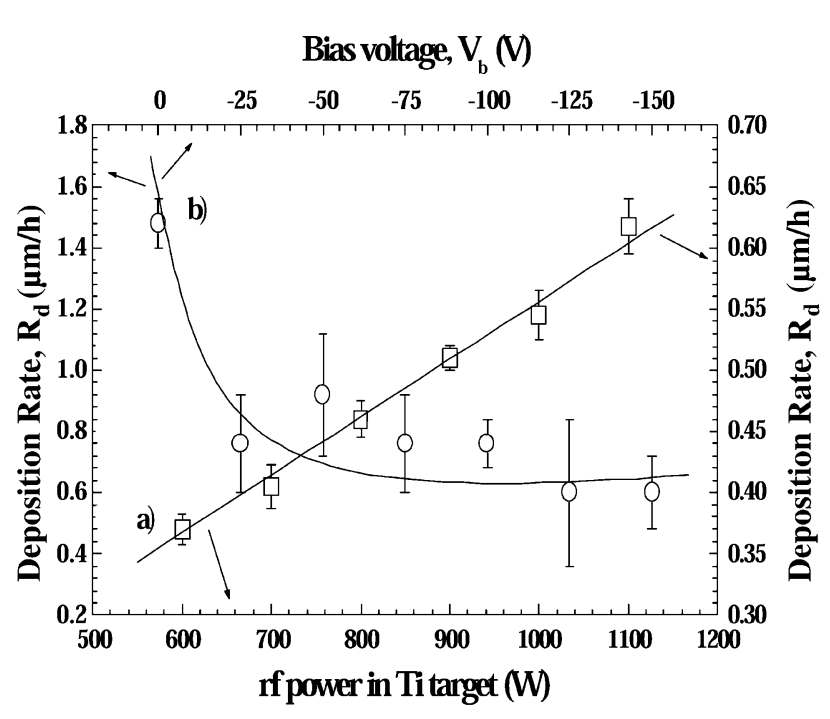

Fig. 1. Deposition rate, $R_{\mathrm{d}}$, of TiN thin films prepared with: (a) variation of the rf power applied in Ti target; and (b) variation of the dc bias voltage applied in the substrate.

substrate surface. Due to the different size of the samples it is not possible to give an accurate quantitative characterization of texture evolution, although a clear reduction in the peak intensity ratio, $T=I_{(111)} / I_{[(200)+(111)]}$ [18], indicates that the increase in the applied power results in less textured films (Fig. 2a). This is a consequence of the increase in the Ti arrival rate at the growing film, which increases the difficulty for structural arrangement [19], changing the preferred crystalline orientation or, in some cases, the appearance of structural defects, intrinsic stresses, etc. [19]. This can also explain the result of the sample prepared with a rf power of $1000 \mathrm{~W}$, which shows a significant shift in the angular position of the peaks $(a=0.426 \mathrm{~nm})$ towards that of stress-free TiN $\left(a_{0}=0.424 \mathrm{~nm}\right.$ [17] $)$. Since at a high deposition rate the surface energy will be no longer the main driving force in the first stages of growth, the accumulation of compressive stresses becomes the dominant process, and thus the occurrence of some stress relieves might explain this shift in peak position. In this sample, a reduced peak width at half maximum (FWHM) is also observed, indicating an increase in grain size. This result is also consistent with the possibility that, beyond stress-relieves, some recrystallisation might occur [10]. Instead, at $900 \mathrm{~W}$ films grow with smaller grains leading to a structure different from that of bulk TiN. There are no significant lattice parameter changes for samples deposited at powers below $900 \mathrm{~W}$, where the value of the lattice parameter is close to 0.430 nm. Peak profiling by Fourier analysis revealed a decrease in grain size from approximately $9 \mathrm{~nm}$ at 600 $\mathrm{W}$ to $6 \mathrm{~nm}$ for powers up to $900 \mathrm{~W}$. For higher powers the existence of TiN grains much higher than $10 \mathrm{~nm}$ is observed. The preferred recrystallisation at these higher powers, promoted by the referred increase in the arrival ratio of deposition material, could explain this result.

SEM images (Fig. 3) revealed that the films grow with a columnar-type structure, lying in the transition between the $\mathrm{T}$ and I zones of Thornton Model [20]. There are no significant changes in the morphology with increasing power as shown in Fig. 3. Plan view observation is also consistent with this fact. However, there is an increase of the surface defects with increasing $\mathrm{rf}$
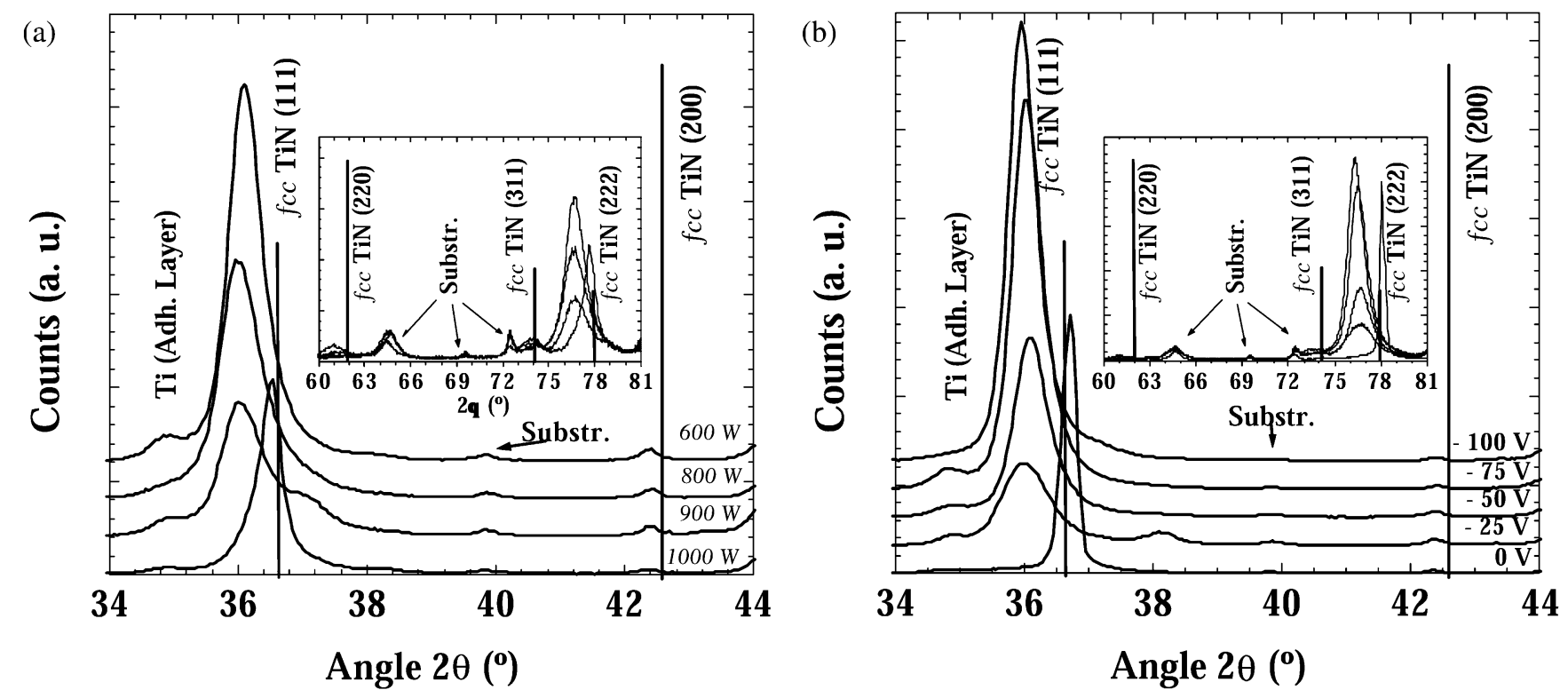

Fig. 2. XRD diffraction patterns of TiN films prepared at: (a) constant bias voltage $(-50 \mathrm{~V})$ and different powers at the Ti target $(600$, 800 , 900 and $1000 \mathrm{~W})$; and (b) constant rf power $(600 \mathrm{~W})$ and variation in bias voltage: (a) $0,-25,-50,-75$ and $-100 \mathrm{~V}$. 

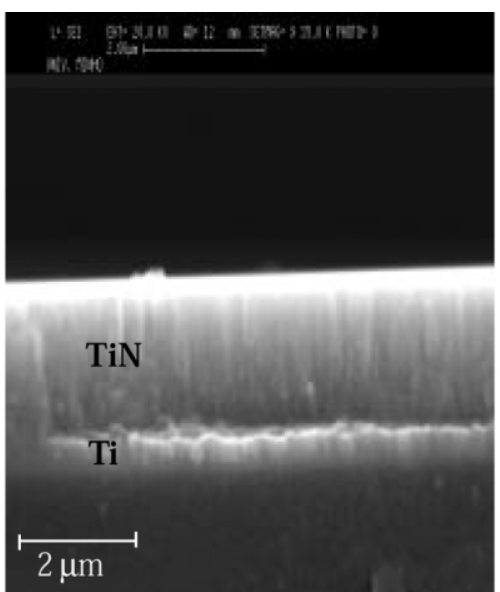

(a)

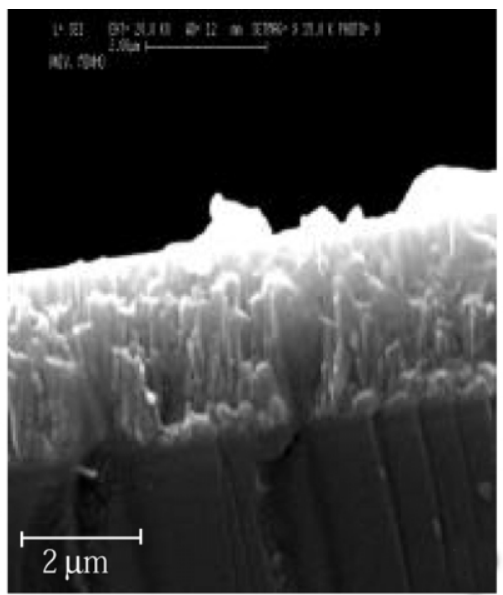

(b)

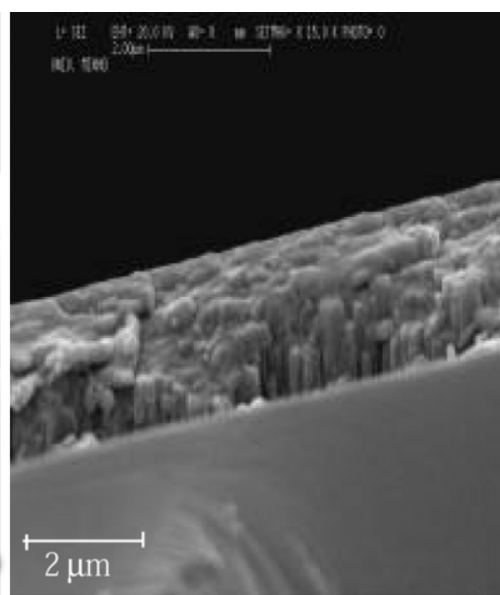

(c)

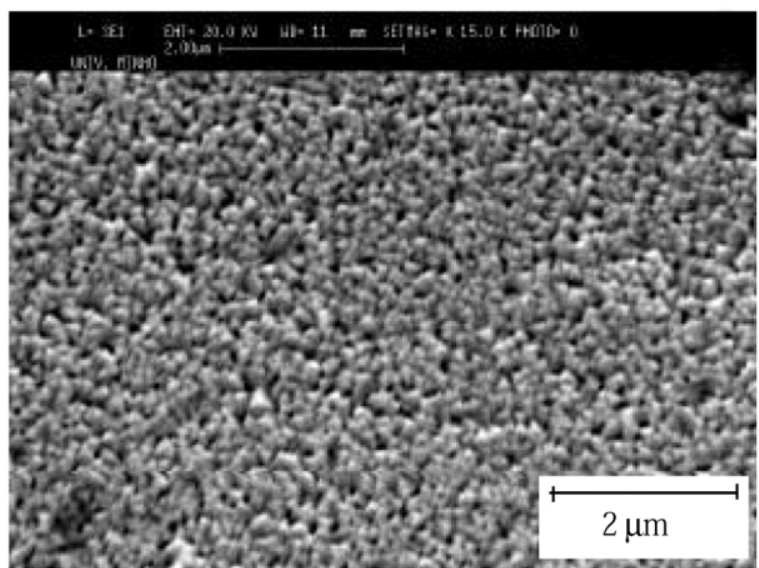

(d)

Fig. 3. SEM cross-section images showing the morphological changes in TiN thin films prepared with constant bias voltage ( $-50 \mathrm{~V})$ and with: (a) $600 \mathrm{~W}$; (b) $700 \mathrm{~W}$; and (c) $800 \mathrm{~W}$ rf power at the Ti target. The micrograph in the bottom (d), shows a plan view image of the sample prepared at $700 \mathrm{~W}$.

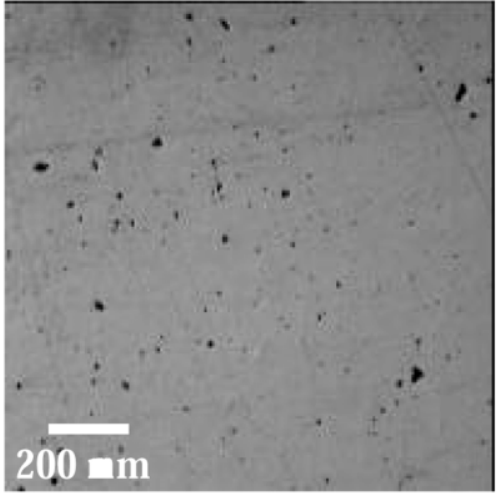

(a)

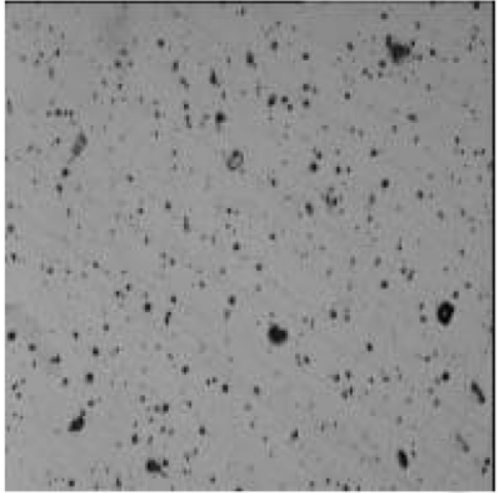

(b)

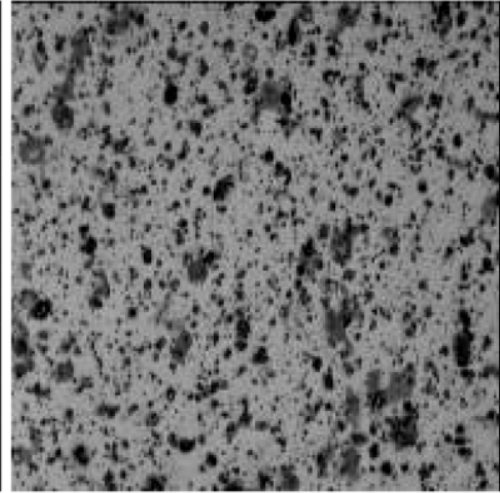

(c)

Fig. 4. OM images of the surface for TiN films prepared at constant bias voltage ( $-50 \mathrm{~V}$ ) and (a) $600 \mathrm{~W}$, (b) $800 \mathrm{~W}$ and (c) $1100 \mathrm{~W}$. 


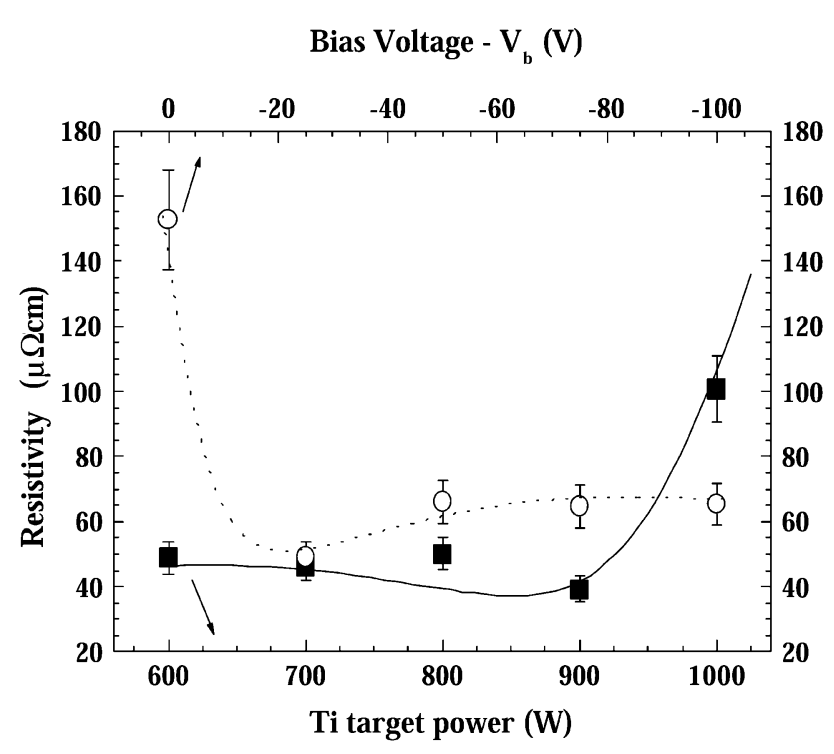

Fig. 5. Electric resistivity, $\rho$, of TiN films prepared at: (a) constant bias voltage $(-50 \mathrm{~V})$ and different powers at the Ti target $(600,800$, 900 and $1000 \mathrm{~W})$; and (b) a constant rf power $(600 \mathrm{~W})$ and variation in bias voltage $(0,-25,-50,-75$ and $-100 \mathrm{~V})$. The lines are guide for the eye.

power, as can be evidenced by OM (Fig. 4). The increase in the arriving species, and the increase in the ion bombardment-supersaturation — are the two main parameters explaining this behaviour.

The films used for resistivity characterisation where deposited on Si substrates. Due to the high range of the silicon resistivity and to the $\mathrm{Ti}$ adhesion layer, the values obtained in the direct measurements have contributions from both. To extract the real resistivity of the TiN films, the values of the ensemble (substrate + adhesion layer) were measured, and a simple model of parallel resistances was assumed. Fig. 5a shows the extracted room temperature resistivity of the TiN films with increasing $\mathrm{rf}$ power. The resistivity of these films is almost constant and close to $60 \mu \Omega \mathrm{cm}$, for powers below $900 \mathrm{~W}$. The high resistivity value obtained for the film deposited at $1000 \mathrm{~W}(\sim 215 \mu \Omega \mathrm{cm})$ is probably due to cracks associated with the stressrelieves, usually observed in films deposited at high powers, which develop high compressive stresses [21,22].

Fig. 6 shows the variation of the colour co-ordinates across a particular sample. The upper left inset shows the sample surface and the analysed area. Fig. 7a shows the average colour for each sample. Error bars represent $\pm 1 / 2$ standard error of the mean (S.E.M.) across the analysed area (see Fig. 6). Colour variations within and across samples were small (less than five units in the CIELAB 1976 space), showing a significant degree of uniformity, which is consistent with the non-significant structural changes that were observed. Nevertheless, there seems to be a slight shift in the $a^{*}$ value (red: positive axis-green: negative axis) for the $600 \mathrm{~W}$ sample, and a slight increase in the $b^{*}$ value (yellow: positive axis-blue: negative axis) with increasing power, which could be a consequence of species arrival ratio [4] (smaller in the $600 \mathrm{~W}$ case), and also to small local variations in the atomic composition [4,9]. Anyway, the similarity of lightness values, $L^{*}$, is consistent with the existence of films with concentration close to the stoichiometric case $[4,9]$.

\subsection{Dependence on the applied bias voltage}

The influence of the bias voltage on the deposition rate is shown in Fig. 1b. The deposition rate decreases sharply with increasing negative bias voltages, $V_{\mathrm{b}}$, in the range 0 to $-25 \mathrm{~V}$. The rapid decrease in the deposition rate for negatively biased experiments is a consequence of the increase in the bias current, $I_{\mathrm{s}}$, promoted by the ion bombardment of the growing films. Measurement of the bias current showed that $I_{\mathrm{s}}$ increases rapidly for bias voltages in the range 0 to $-25 \mathrm{~V}$, reaching a significant value very close to the stationary regime [23]. In fact, the great similarity on the deposition rates throughout the negative biased samples is the result of this ion current at the substrate, which at -25 $\mathrm{V}$ is already as much as that at $-50 \mathrm{~V}$, being then almost constant for the other negative bias values. Furthermore, this change in the deposition rate is also correlated with changes in film morphology. This bombardment causes re-sputtering of deposited species, decreasing the deposition rate [10].

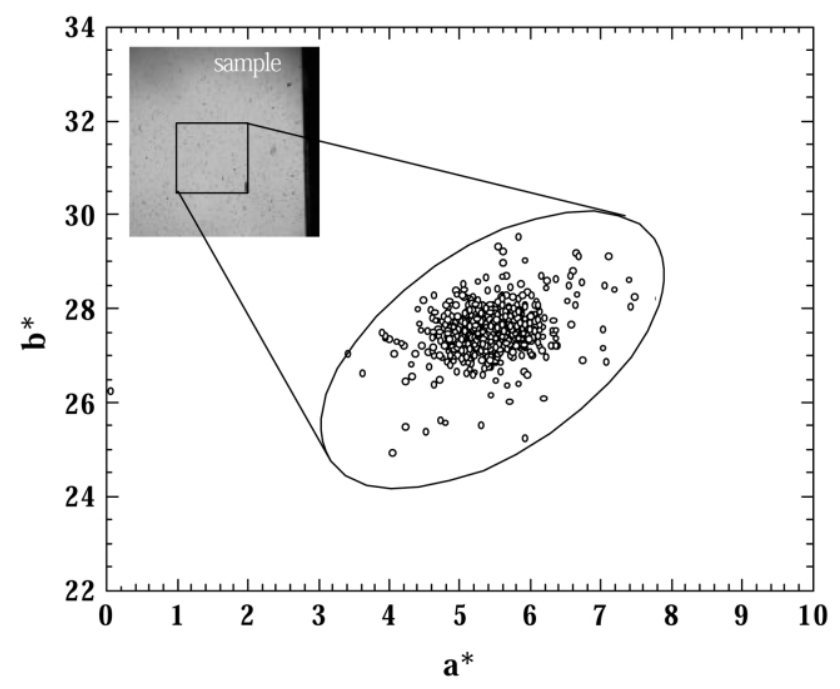

Fig. 6. Variation of colour in the CIELAB 1976 colour space $[14,15]$ across a particular TiN sample $(700 \mathrm{~W} ;-50 \mathrm{~V})$. The upper left inset shows the sample surface and the analysed area with $3.7 \times 3.7 \mathrm{~mm}$ and a spatial resolution of $12 \mu \mathrm{m} /$ pixel. 

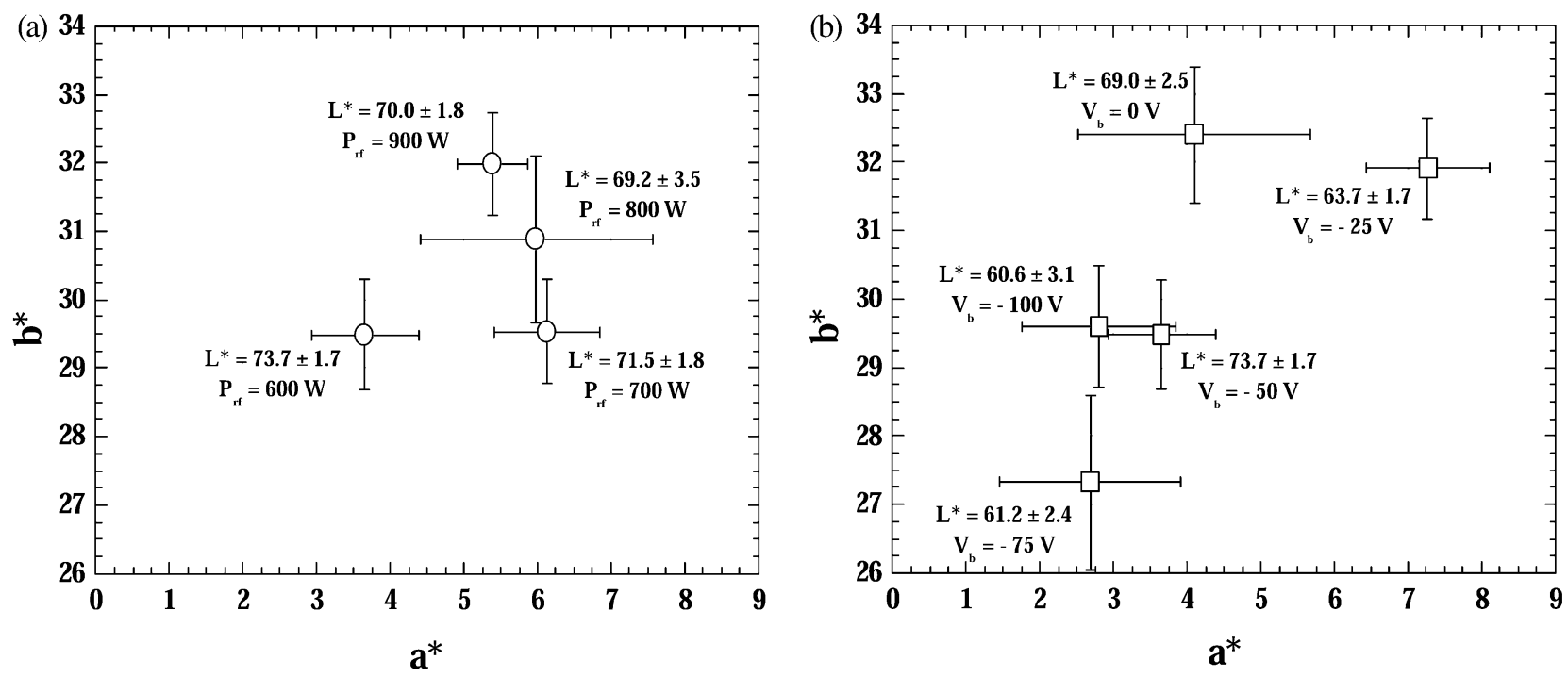

Fig. 7. Average specular colour in the CIELAB 1976 colour space for the samples under the standard CIE illuminant $\mathrm{D}_{65}$ and prepared with: (a) different rf powers at the target; and (b) different bias voltages. Error bars represent $\pm 1 / 2$ standard error of the mean (S.E.M.) across an area of $3.7 \times 3.7 \mathrm{~mm}$ analysed with a spatial resolution of $12 \mu \mathrm{m} /$ pixel.

X-Ray diffraction experiments show the $\delta$-TiN phase [17] for all the samples of this set. Additional diffraction peaks from steel substrates and the Ti adhesion (buffer) layer are also clear. The increase in the bias voltage seems to favour the texture effect, as is evidenced by the increase in the peaks intensity ratio, $T=I_{(111)} /$ $I_{[(200)+(111)]}$, Fig. 2b. This observation is consistent with the increase in the deformation energy, promoted by the ion bombardment [24], which becomes the main driving force, favouring the [111] growth. As $\left|V_{\mathrm{b}}\right|$ increases, the kinetic energy of the impinging ions and atoms increase, resulting in enhanced surface mobility and the development of a fine-grained structure. In addition, defects induced by the impinging $\mathrm{Ar}^{+}$can serve as secondary nucleation sites [25] and along with the enhanced adatom mobility can result in grain refinement. Moreover, by increasing adatom mobility, shadowing effects are eliminated and the porosity fraction in the films is decreased leading to denser films [26]. Another significant aspect, which shows the influence of the ion bombardment, is the evolution of grain size. The XRD result of the non-biased sample (absence of ion bombardment) is notorious, since both peak position and FWHM do not match with the rest of the samples. The simulation of the (111) peaks revealed an increase in grain size from approximately $8 \mathrm{~nm}\left(\right.$ at $\left.V_{\mathrm{b}}=-25 \mathrm{~V}\right)$ to $9 \mathrm{~nm}$ (for $\mathrm{V}_{\mathrm{b}}=-50 \mathrm{~V}$ ), and approximately $10 \mathrm{~nm}$ for the higher bias voltages $\left(V_{\mathrm{b}}=-75\right.$ and $\left.-100 \mathrm{~V}\right)$, while the simulation in the grounded sample resulted in grains larger than $12 \mathrm{~nm}$. The absence of ion bombardment, together with the relative low target power allows the grain growth, being the most probable factor that explains this behaviour. Anyway, the grain refinement is very reduced in this sample, leading to a large number of defects. These characteristics will severely influence the film properties [21]. Regarding the lattice parameters, calculations show a behaviour very similar to that of the other set of samples, with no significant variations and a value of approximately $0.430 \mathrm{~nm}$, except for the grounded sample that has lattice parameter close to the bulk value $(0.424 \mathrm{~nm})$, which is a consequence of the already mentioned more unconstrained grain growth.

Fig. 8 shows SEM images revealing typical morphology of the TiN films deposited with variation in the bias voltage applied to the substrate. As it can be seen, the films are also growing with a columnar-type structure, lying in the T and I zone of Thornton Model [20]. In contrast to the results obtained for the other set of films, there is a clear tendency for the densification of the structure with the ion bombardment. By comparison of the different cross-section SEM images in Fig. 8, it is worth noticing that the columnar growth in the unbiased sample is less dense and different from the biased samples. Furthermore, the surface images support this idea, showing a roughness increase with the negative bias voltage. The surface defects evolution of the samples observed by OM reveals again a clear tendency for an increase of surface defects with the increase of negative bias voltage. The increase in the ion bombardment is the main factor that explains this behaviour.

The electric resistivity of this set of samples is shown in Fig. 5b. The values observed are similar to the other set of samples $(\sim 60 \mu \Omega \mathrm{cm})$ with the exception of the grounded sample, which has a resistivity of $\sim 153 \mu \Omega$ 


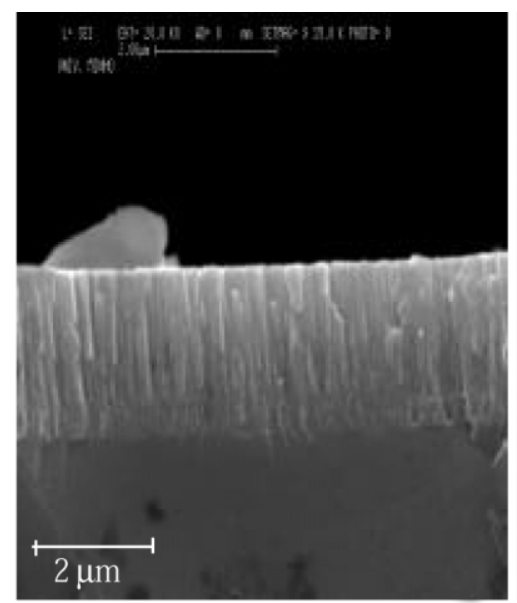

(a)

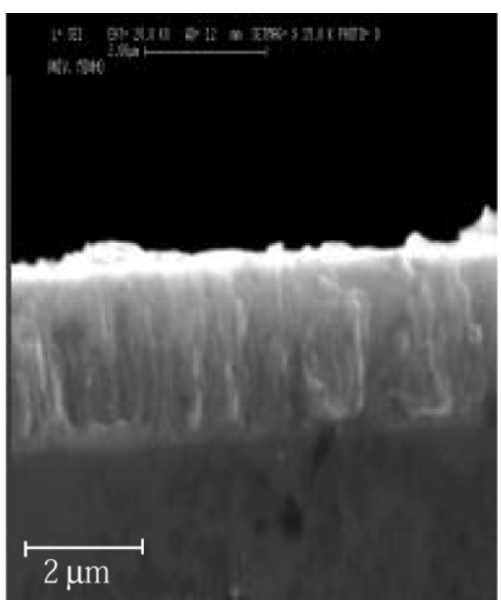

(b)

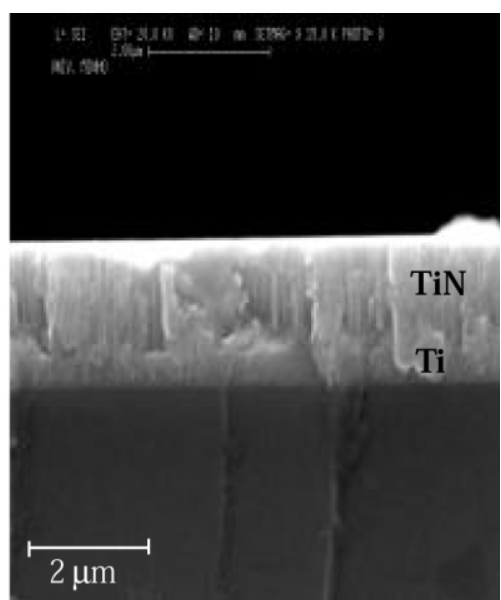

(c)

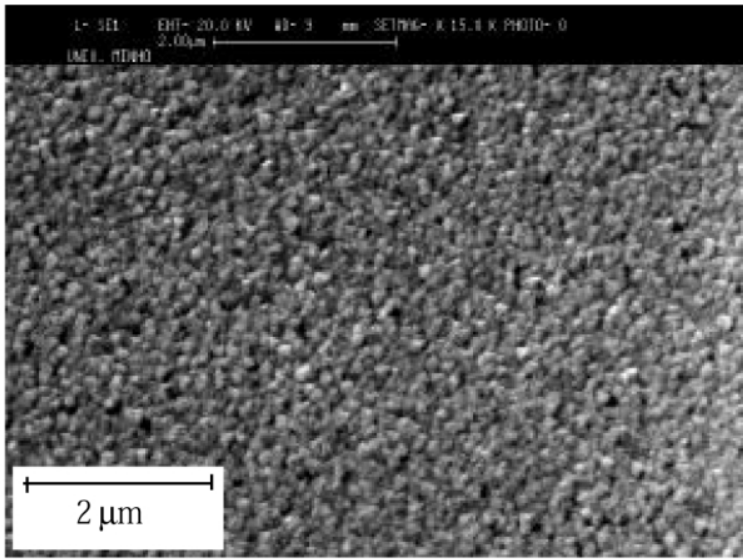

(d)

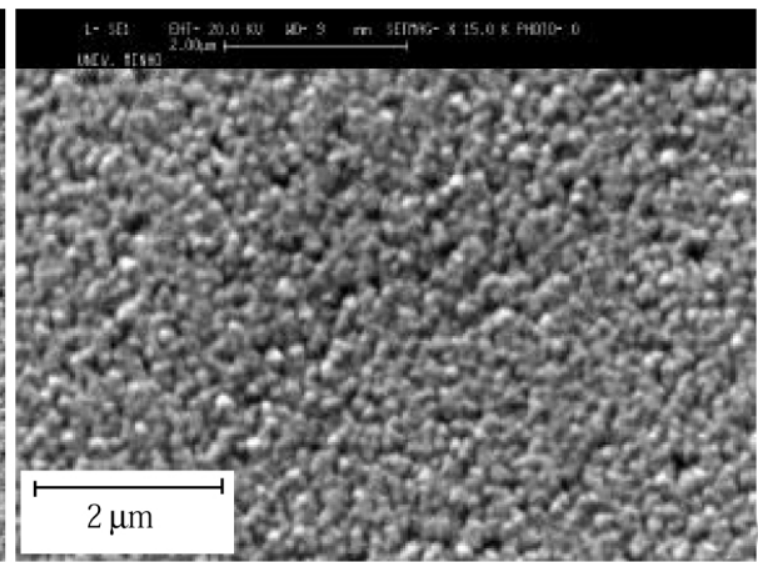

(e)

Fig. 8. Above: SEM cross-section images showing the morphological changes in TiN thin films prepared with constant rf power (600 W) and under: (a) 0 (grounded); (b) $-25 \mathrm{~V}$; and (c) $-100 \mathrm{~V}$ dc bias voltages. Below: these two micrographs represent plan view images of the sample prepared: (d) without [grounded sample illustrated in (a) ] and (e) with [ $-25 \mathrm{~V}$ bias voltage illustrated in (b)] ion bombardment of the growing films.

$\mathrm{cm}$. This higher value is again a consequence of the lack of ion bombardment that leads to less dense films and to a higher number of defects.

Fig. $7 \mathrm{~b}$ shows the average colour for each of the samples, prepared with variations in bias voltage. Colour variations within and across samples were larger than for the previous set of samples and lightness was, on average, lower. The ion bombardment and some small variations in composition, promoted by the selective resputtering [10] might be responsible for this higher scattering. It is worth noticing, however, that the unbiased sample although showing a comparatively higher resistivity, retained the golden colour. In literature there are a few works that report TiN films having a golden colour and low resistivity with the no-bias condition [27-29]. These contradictory results indicate that the dependence of the film properties on the sputtering conditions and the criterion for colour selection are still unclear. Moreover, the results of this paper emphasises that the relationship between electrical resistivity and the colour of TiN films is far from being restricted to the applied bias as suggested by other works $[6,7,30]$. In fact, microstructure is a fundamental issue for these properties as suggested by Nose et al. [9]. Although not very clear, the texture phenomena (preferential growth, among others) together with residual stress states, and also the different morphological aspects, such as density, defects, etc., play an important role in the variation of both properties. The fact that no significant changes in crystal growth were observed in these films is consistent with the small variations in colour measurements. However, the accumulation of residual stresses (samples prepared at high powers) and defects resulting from the low adatom mobility (in the unbiased sample), together 
with the grain growth are the main parameters that might rule resistivity, but not colour.

\section{Conclusion}

In conclusion, the changes in deposition rates due to rf power and ion bombardment of the substrate produce films with different structural and physical properties. The increase in film density due to the decrease of voids between the crystallites is promoted by the ion bombardment. Also the re-sputtering of the growing film by the argon ions could induce the selective removal of impurities, such as oxygen and argon from the film.

$\mathrm{X}$-Ray diffraction experiments show that there is a unique $\delta$-TiN phase with fcc, NaCl-type lattice for all the samples. The lattice parameter for all samples deposited at powers below $1000 \mathrm{~W}$ and negative bias voltage is approximately $0.430 \mathrm{~nm}$. The sample deposited at $1000 \mathrm{~W}$ has a lattice parameter of $0.426 \mathrm{~nm}$, close to that of the stress-free material $\left(a_{0}=0.424 \mathrm{~nm}\right)$. The grounded sample also reveals a lattice parameter close to the bulk value $(0.424 \mathrm{~nm})$, due to the low stresses that this sample develops.

All films grow with a columnar-type structure, lying in the $\mathrm{T}$ and I zone of Thornton Model. There are no significant changes in the morphology with increasing power and bias voltage. In the unbiased sample, the columns are bettered defined and less dense than in the negatively biased samples, which explain the variation in resistivity. The resistivity of the TiN films is almost constant and close to $60 \mu \Omega \mathrm{cm}$, for powers below $1000 \mathrm{~W}$ and negatively biased. The high resistivity value $(\sim 215 \mu \Omega \mathrm{cm})$ observed for the film deposited at $1000 \mathrm{~W}$ is probably due to cracks associated with stress-relieves.

No significant colour changes were observed for the different conditions of deposition. From the discussion above, it might be concluded that the electrical properties of TiN depend critically on the microstructural and morphological aspects and hence on the growth conditions, such as the growing species mobility and evolution of compressive stresses. The optical properties do not seem to depend as much on these structural and morphological issues as on the composition itself.

\section{References}

[1] D.A. Glocker, S.I. Shah, Handbook of Thin Film Process Technology, 2, IOP Publishing, Bristol and Philadelphia, 1995, and references therein
[2] H. Randhawa, Surf. Coat. Technol. and references therein 36 (1988) 829.

[3] J. Rivory, J.M. Behaghel, S. Berthier, J. Lafait, Thin Solid Films 78 (1981) 161.

[4] U. Beck, G. Reiners, U. Kopacz, H.A. Jehn, Surf. Coat. Technol. 60 (1993) 389.

[5] V. Valvoda, R. Kuzel, R. Cerany, J. Musil, Thin Solid Films 156 (1988) 53.

[6] A. Mumtaz, W.H. Class, J. Vac. Sci. Technol. 20 (3) (1982) 345.

[7] N. Kumar, J.T. McGinn, K. Pourrezaei, B. Lee, E.C. Douglas, J. Vac. Sci. Technol. A 6 (3) (1988) 1602.

[8] R. Manory, Surf. Eng. 3 (3) (1987) 233.

[9] M. Nose, T. Nagae, M. Yokota, S. Saji, M. Zhou, M. Nakada, Surf. Coat. Technol. 116-119 (1999) 296.

[10] M. Ohring, The Materials Science of Thin Films, Academic Press, San Diego, 1992.

[11] F. Vaz, Minho University, Portugal, January 2002, personal communication.

[12] J. Mignot, S. Rondot, Acta Metall. 23 (1975) 1321.

[13] Van der Pauw, Philips Research Reports 16 (1961) 187.

[14] Comission Internationale de L'Éclairage, Colorimetry, CIE Publication 15, 1971.

[15] Comission Internationale de L'Éclairage, Recommendations on Uniform Colour Spaces, Colour-difference Equations, Psychometric Colour Terms, CIE Publication 15 Suppl. No. 2-70, 1978.

[16] P.Y. Jouan, G. Lempérère, Thin Solid Films 237 (1994) 200.

[17] Powder Diffraction File of the International Center for Diffraction, PDF-ICDD Card No. 38-1420

[18] S. Groudeva-Zotova, R. Kaltofen, T. Sebald, Surf. Coat. Technol. 127 (2000) 144.

[19] Y. Imai, M. Mukaida, A. Watanabe, T. Tsunoda, Thin Solid Films 300 (1997) 305, See for an example of a more detailed analysis of this behavior.

[20] J.A. Thorton, Am. Rev. Mater. Sci. 7 (1977) 239.

[21] P. Machado, Minho University, Portugal, July 2001, personal communication.

[22] V. Branger, C. Coupeau, Ph. Goudeau, B. Boubeker, K.F. Badawi, J. Grilhé, J. Mater. Sci. Lett. 19 (2000) 353.

[23] E. Ribeiro, A. Malczyk, S. Carvalho, L. Rebouta, J.V. Fernandes, E. Alves, A.S. Miranda, Surf. Coat. Technol. 151/152 (2002) 515.

[24] J. Pelleg, L.Z. Zevin, S. Lungo, Thin Solid Films 197 (1991) 117.

[25] J.E. Sundgren, Thin Solid Films 128 (1985) 21.

[26] J.M. Poitevin, G. Lemperiere, J. Tardy, Thin Solid Films 97 (1982) 69.

[27] R. Manory, Surf. Eng. 3 (3) (1987) 233.

[28] H.Q. Lou, N. Axen, R.E. Somekh, I.M. Hutchings, Surf. Coat. Technol. 90 (1997) 123.

[29] M. Kawamura, Y. Abe, H. Yanagisawa, K. Sasaki, Thin Solid Films 287 (1996) 115.

[30] S. Kanamory, Thin Solid Films 136 (1986) 195. 